Check for updates

For numbered affiliations see

end of the article.

Correspondence to: P Ueda

peter.ueda@ki.se

(or @PeterUeda1 on Twitter

ORCID 0000-0002-3275-8743)

Additional material is published online only. To view please visit

the journal online.

Cite this as: $B M J 2020 ; 369: \mathrm{m} 1186$ http://dx.doi.org/10.1136/bmj.m1186

Accepted: 13 March 2020
OPEN ACCESS

\section{Use of sodium-glucose co-transporter 2 inhibitors and risk of serious renal events: Scandinavian cohort study}

\author{
Björn Pasternak, ${ }^{1,2}$ Viktor Wintzell, ${ }^{1}$ Mads Melbye, ${ }^{2,3,4}$ Björn Eliasson, ${ }^{5}$ Ann-Marie Svensson, ${ }^{5,6}$ \\ Stefan Franzén,, ${ }^{6,7}$ Soffia Gudbjörnsdottir, ${ }^{5,6}$ Kristian Hveem, ${ }^{8,9}$ Christian Jonasson, ,,9,10 \\ Henrik Svanström, ${ }^{1,2}$ Peter Ueda ${ }^{1}$
}

\section{ABSTRACT \\ OBJECTIVE}

To assess the association between use of sodiumglucose co-transporter 2 (SGLT2) inhibitors and risk of serious renal events in data from routine clinical practice.

DESIGN

Cohort study using an active comparator, new user design and nationwide register data.

SETTING

Sweden, Denmark, and Norway, 2013-18.

PARTICIPANTS

Cohort of 29887 new users of SGLT2 inhibitors (follow-up time: dapagliflozin 66.1\%; empagliflozin $32.6 \%$; canagliflozin $1.3 \%$ ) and 29887 new users of an active comparator, dipeptidyl peptidase- 4 inhibitors, matched 1:1 on the basis of a propensity score with 57 variables. Mean follow-up time was 1.7 (SD 1.0) years.

EXPOSURES

SGLT2 inhibitors versus dipeptidyl peptidase-4 inhibitors, defined by filled prescriptions and analysed according to intention to treat.

\section{MAIN OUTCOME MEASURES}

The main outcome was serious renal events, a composite including renal replacement therapy, death from renal causes, and hospital admission for renal events. Secondary outcomes were the individual components of the main outcome.

RESULTS

The mean age of the study population was 61.3 (SD 10.5) years; 11108 (19\%) had cardiovascular disease, and 1974 (3\%) had chronic kidney disease. Use of SGLT2 inhibitors, compared with dipeptidyl

\section{WHAT IS ALREADY KNOWN ON THIS TOPIC}

Clinical trials have shown that sodium-glucose co-transporter 2 (SGLT2) inhibitors protect renal function among patients with type 2 diabetes and high cardiovascular risk or established nephropathy

The effect of SGLT2 inhibitors on serious renal events in broader unselected groups of patients in routine clinical practice remains uncertain

\section{WHAT THIS STUDY ADDS}

This cohort study used nationwide register data from Sweden, Denmark, and Norway to compare use of SGLT2 inhibitors and dipeptidyl peptidase- 4 inhibitors Use of SGLT2 inhibitors was associated with a $58 \%$ lower risk of a composite outcome of serious renal events, including renal replacement therapy, death from renal causes, and hospital admission for renal events

Complementing the results of randomised trials, these data suggest that SGLT2 inhibitors may lower the risk of serious renal events in routine clinical practice peptidase- 4 inhibitors, was associated with a reduced risk of serious renal events (2.6 events per 1000 person years versus 6.2 events per 1000 person years; hazard ratio 0.42 (95\% confidence interval 0.34 to 0.53$)$; absolute difference $-3.6(-4.4$ to -2.8$)$ events per 1000 person years). In secondary outcome analyses, the hazard ratio for use of SGLT2 inhibitors versus dipeptidyl peptidase- 4 inhibitors was 0.32 (0.22 to 0.47$)$ for renal replacement therapy, 0.41 (0.32 to 0.52 ) for hospital admission for renal events, and 0.77 ( 0.26 to 2.23 ) for death from renal causes. In sensitivity analyses in each of the Swedish and Danish parts of the cohort, the model was further adjusted for glycated haemoglobin and estimated glomerular filtration rate (Sweden and Denmark) and for blood pressure, body mass index, and smoking (Sweden only); in these 0.66 ) to 0.50 ( 0.31 to 0.81 ) in Sweden and from 0.42 (0.32 to 0.56 ) to 0.55 (0.41 to 0.74 ) in Denmark.

\section{CONCLUSIONS}

In this analysis using nationwide data from three countries, use of SGLT2 inhibitors, compared with dipeptidyl peptidase-4 inhibitors, was associated with a significantly reduced risk of serious renal events.

\section{Introduction}

Type 2 diabetes is the leading cause of kidney failure. ${ }^{1}$ Although treatment with angiotensin converting enzyme inhibitors and angiotensin receptor blockers reduces the risk of adverse renal outcomes in patients with diabetes, ${ }^{2-4}$ the risk remains high and a large need exists for new treatments that lower the risk of kidney failure. Sodium-glucose co-transporter 2 (SGLT2) inhibitors are a class of glucose lowering drugs that also reduce blood pressure, body weight, and albuminuria. Large clinical trials have shown that these drugs have beneficial effects on renal outcomes. In the CREDENCE trial, patients with type 2 diabetes and albuminuric chronic kidney disease who received canagliflozin experienced lower rates of the composite renal outcome versus placebo, including end stage kidney disease, a doubling of serum creatinine concentration, and death from renal causes (hazard ratio $0.66,95 \%$ confidence interval 0.53 to 0.81$).^{5}$ Similarly, rates of composite renal outcomes were reduced among patients with type 2 diabetes and high cardiovascular risk receiving canagliflozin in the CANVAS programme (hazard ratio $0.58,0.50$ to 0.67$),{ }^{6}$ empagliflozin in the EMPAREG OUTCOME trial (0.61, 0.53 to 0.70$),{ }^{7}$ and dapagliflozin in the DECLARE TIMI-58 trial $(0.53,0.43$ to 0.66$){ }^{8}$

The data from clinical trials provide evidence for the renoprotective effects of SGLT2 inhibitors, but analyses, the hazard ratio moved from 0.41 ( 0.26 to 
uncertainty remains about the effect of these drugs on renal outcomes in routine clinical practice. The four large clinical trials assessing renal outcomes with SGLT2 inhibitors have included only patients at high cardiovascular risk or with established nephropathy. ${ }^{5-8}$ Patients receiving SGLT2 inhibitors in clinical practice tend to be more heterogenous, ${ }^{9}$ and whether the findings of the clinical trials are generalisable to broad, unselected groups of patients is unknown.

Using nationwide data from patients seen in routine clinical practice in Sweden, Denmark, and Norway, we did a register based cohort study to assess whether use of SGLT2 inhibitors, compared with an active comparator (dipeptidyl peptidase-4 inhibitors), is associated with a reduced risk of serious renal events.

\section{Methods}

\section{Data sources}

Weused data from nationwidehealth and administrative registers in Sweden (April 2013 through December 2016), Denmark (April 2013 through December 2018), and Norway (April 2013 through December 2016). Data sources included population registers and Statistics Denmark/Statistics Sweden (vital status, demographics, socioeconomic variables), patient registers (comorbidities, outcomes), prescription registers (study drugs, co-medications), cause of death registers (outcomes; data from this register in Denmark were available through 2017), the Swedish National Diabetes Register (glycated haemoglobin level, blood pressure, albuminuria, estimated glomerular filtration rate, body mass index, and smoking), and the Danish Register of Laboratory Results for Research (glycated haemoglobin, albuminuria, and estimated glomerular filtration rate); details are provided in the supplementary material.

\section{Active comparator, new user design}

We used an active comparator, new user design to mitigate the risk of confounding by indication, disease severity, and unmeasured clinical characteristics. ${ }^{10}$ The ideal active comparator would be a drug that is used in similar clinical situations to SGLT2 inhibitors and has no expected associations with the investigated outcomes. We used dipeptidyl peptidase-4 inhibitors as the active comparator, as clinical guidelines used during the study period recommended both SGLT2 inhibitors and dipeptidyl peptidase-4 inhibitors as second line or third line glucose lowering therapies and data from clinical trials in patients at high cardiovascular risk indicate that dipeptidyl peptidase-4 inhibitors have no or limited effects on renal outcomes. ${ }^{1112}$

\section{Study population}

We included all patients aged 35-84 years in the three countries who filled their first prescription for either an SGLT2 inhibitor or a dipeptidyl peptidase-4 inhibitor during the study period (anatomic therapeutic chemical codes for study drugs are shown in supplementary table A). The date of filling the first prescription constituted cohort entry. Exclusion criteria were previously filled prescriptions for any of the study drugs within two years of cohort entry, no specialist care contact or prescription drug in the previous year, history of dialysis or renal transplantation, end stage illness, drug misuse, severe pancreatic disorders, and hospital admission for any reason within 30 days before cohort entry (supplementary table B).

Using logistic regression, we estimated the probability of starting a SGLT2 inhibitor versus a dipeptidyl peptidase-4 inhibitor, conditional on the status of 57 covariates at cohort entry; the score included variables on sociodemographic characteristics, comorbidities, co-medications, and healthcare utilisation (supplementary table C). We used missing categories to handle missing data on place of birth $(<1 \%)$, civil status $(<1 \%)$, and education $(<3 \%)^{13}$; none of the other variables had missing data. We estimated propensity scores in each country separately. Owing to variations in data availability, a few variables included in the propensity score in Norway differed slightly from those in Sweden and Denmark (supplementary table C).

We matched new users of SGLT2 inhibitors and dipeptidyl peptidase- 4 inhibitors (1:1 ratio) by using the nearest neighbour algorithm (calliper width 0.2 of the standard deviation of the logit propensity score), ${ }^{1415}$ with sex, age ( 5 year intervals), and a previous diagnosis of chronic kidney disease (supplementary table D) as additional matching criteria. We considered covariates to be well balanced if the standardised difference was below $10 \%$. We did the analyses in a pooled dataset of the matched cohorts of the three countries.

\section{Outcomes}

The primary outcome, serious renal events, was a composite of renal replacement therapy (dialysis or renal transplantation), death from renal causes, and hospital admission for renal events, as captured in the patient registers and the cause of death registers. Secondary outcomes were each component of the primary outcome.

Hospital admission for renal events was based on events consistent with serious renal disease, including diabetic nephropathy, chronic kidney disease, and acute kidney injury; we considered this outcome as a renal analogue to the outcome of hospital admission for heart failure in cardiology, in that we regarded it as an indicator of serious worsening of renal status. Supplementary table E shows ICD-10 (international classification of diseases, version 10) codes and procedure codes used to define the outcomes. In validation studies of diagnostic codes for renal events used in health registers, sensitivity has varied widely, whereas specificity was high. ${ }^{16}$ Validation studies have not been done in the Scandinavian setting and for the specific codes used in our analyses.

\section{Statistical analyses}

We followed patients from cohort entry until outcome event, death, emigration, three years of follow-up, or 
the end of the study period. Patients were censored at the time of the first outcome event in the analyses of the primary outcome; in analyses of secondary outcomes, patients were censored at the first occurrence of the outcome analysed, independent of other outcomes. We used an intention to treat exposure definition in which patients were defined as being exposed to the study drug from cohort entry throughout follow-up. We used Cox proportional hazards regression with time since cohort entry as the time scale to estimate hazard ratios. We examined the assumption of proportional hazards by using a Wald test of the interaction between treatment status and time. We considered hazard ratios with $95 \%$ confidence intervals that did not overlap 1 to be statistically significant. We estimated the absolute rate difference assuming a Poisson distribution.

For the primary outcome, we did subgroup analyses by sex, age group, history of major cardiovascular disease (supplementary table F), and history of chronic kidney disease. We used an interaction term between treatment status and subgroup to assess effect modification by subgroup status; in these analyses, we considered a P value below 0.05 to be statistically significant. We also analysed the primary outcome by country to assess consistency across data sources and in separate analyses for patients starting empagliflozin and dapagliflozin (and their 1:1 matched users of dipeptidyl peptidase-4 inhibitors), respectively; this analysis was not possible for canagliflozin, as few patients used this drug.

In an additional analysis, we used an as-treated exposure definition based on the estimated duration of the filled prescriptions (supplementary table A), allowing for a 30 day grace period to account for prescription overlap, irregular drug use, and events that occurred shortly after treatment cessation. In these analyses, patients were censored at treatment cessation and crossover to the other study drug (that is, start of dipeptidyl peptidase-4 inhibitors among users of SGLT2 inhibitors and vice versa).

We did several sensitivity analyses. Firstly, we adjusted the analysis of the primary outcome for calendar year of cohort entry. Next, in analyses in each of the propensity score matched cohorts in Sweden and Denmark, we adjusted the Cox models for additional variables. In Sweden, these variables included glycated haemoglobin level, blood pressure, albuminuria, estimated glomerular filtration rate, body mass index, and smoking; in Denmark, they included glycated haemoglobin level, albuminuria, and estimated glomerular filtration rate (supplementary table G). Owing to the proportion of patients with missing data for these variables (supplementary table $\mathrm{G}$ ), we used multiple imputation (fully conditional specification imputation) to handle missing data and used 10 imputed datasets for the analyses. ${ }^{17}$ On the basis of the imputed datasets, we also did the analyses in subgroups of patients by level of estimated glomerular filtration rate $\left(<60 v \geq 60\right.$ per $1.73 \mathrm{~m}^{2}$ body surface area) and the presence of albuminuria. For these analyses, we estimated hazard ratios separately for each subgroup in each country and combined them using meta-analysis assuming fixed effects.

\section{Patient and public involvement}

No patients were involved in setting the research question, nor in the design, conduct, or interpretation of the study. The study is based on anonymised nationwide register data, and no dissemination of results directly to study participants is planned.

\section{Results}

\section{Study population}

In all, 38273 new users of SGLT2 inhibitors and 107854 new users of dipeptidyl peptidase-4 inhibitors fulfilled study eligibility criteria (fig 1). Supplementary table $\mathrm{H}$ shows the baseline characteristics of the cohort before matching, and supplementary tables I-K show the characteristics of the patients by country. After one-to-one matching, the cohort included 29887 new users of SGLT2 inhibitors and 29887 new users of dipeptidyl peptidase-4 inhibitors. Covariates in the two groups were well balanced (table 1). The mean age was 61.3 (SD 10.5) years, 39.3\% were female, $18.6 \%$ had a history of major cardiovascular disease, and $3.3 \%$ had a history of chronic kidney disease. Total follow-up time in the primary analysis was 42632 years (mean 1.4 (1.0) years) among SGLT2 inhibitor users and 58473 years (mean 2.0 (1.0) years) among dipeptidyl peptidase-4 inhibitor users. Mean followup time overall was was 1.7 (SD 1.0) years Of the total follow-up for SGLT2 inhibitors, the proportion of follow-up time by drug started at cohort entry was $66.1 \%$ for dapagliflozin, 32.6\% for empagliflozin, and $1.3 \%$ for canagliflozin. For dipeptidyl peptidase- 4 inhibitors, the proportions were $64.8 \%$ for sitagliptin, $20.0 \%$ for vildagliptin, $10.2 \%$ for linagliptin, $2.8 \%$ for saxagliptin, and $2.2 \%$ for alogliptin (supplementary table L).

\section{Primary and secondary outcomes}

Figure 2 shows the cumulative incidence of the primary composite outcome, serious renal events. Use of SGLT2 inhibitors, compared with dipeptidyl peptidase-4 inhibitors, was associated with a significantly lower risk of serious renal events (incidence rate 2.6 events per 1000 person years versus 6.2 events per 1000 person years; hazard ratio 0.42, 95\% confidence interval 0.34 to 0.53 ) (table 2). When we assessed the proportional hazards assumption, we observed a significant interaction between year of follow-up and exposure to SGLT2 inhibitors $(\mathrm{P}=0.009$; Schoenfeld residuals are shown in supplementary figure $\mathrm{A})$. The association of SGLT2 inhibitors with lower risk of serious renal events was largely driven by the first two years of follow-up (hazard ratio 0.34 (0.25 to 0.47 ) for year 1 after cohort entry, 0.43 (0.30 to 0.64$)$ for year 2 , and 0.74 (0.46 to 1.17) for year 3).

In the analyses of the secondary outcomes, use of SGLT2 inhibitors, compared with dipeptidyl peptidase-4 inhibitors, was associated with a significantly lower risk of renal replacement therapy 
(incidence rate $0.8 v 2.5$ per 1000 person years; hazard ratio $0.32,0.22$ to 0.47$)$ and hospital admission for renal events $(2.0 v 4.9$ per 1000 person years; 0.41, 0.32 to 0.52$)$ but not death from renal causes ( $0.2 v 0.2$ per 1000 person years; $0.77,0.26$ to 2.23 ) (table 2).

\section{Subgroup and additional analyses}

Figure 3 shows subgroup analyses. We observed no significant interaction between use of SGLT2 inhibitors and the primary outcome in analyses by sex and age group. Hazard ratios were lower for patients with a history of cardiovascular disease than for those without $(0.30$ (0.21 to 0.44$)$ versus 0.52 ( 0.40 to 0.67$)$; P for interaction=0.022) and for those with a history of chronic kidney disease versus those without $(0.18$ ( 0.10 to 0.31$)$ versus 0.52 ( 0.41 to 0.65$)$; $\mathrm{P}$ for interaction<0.001). Hazard ratios were consistent across countries (supplementary table M; supplementary figure $\mathrm{B}$ ). The hazard ratio was 0.33 (0.22 to 0.49 ) for patients starting empagliflozin and 0.47 (0.36 to 0.61 ) for those starting dapagliflozin (supplementary table $\mathrm{N}$ ).

In the additional analyses using an as-treated exposure definition, the total follow-up time was 21557 years (mean 0.7 (SD 0.6) years) for users of SGLT2 inhibitors and 27314 years (mean 0.9 (0.8) years) for users of dipeptidyl peptidase- 4 inhibitors. In this analysis, the hazard ratio for the association between use of SGLT2 inhibitors and the primary outcome was 0.30 (0.22 to 0.42) (supplementary figure C; supplementary table 0 ) The assumption of proportional hazards was met $(\mathrm{P}=0.69)$ (supplementary figure D).

\section{Sensitivity analyses}

In the analysis adjusted for calendar year of cohort entry, the hazard ratio was unchanged from the primary analyses $(0.42,0.34$ to 0.52$)$. The distribution of glycated haemoglobin, blood pressure, albuminuria, estimated glomerular filtration rate, body mass index, and smoking in the Swedish part of the cohort is shown in supplementary table $\mathrm{P}$, and the distribution of glycated haemoglobin, albuminuria, and estimated glomerular filtration rate in the Danish part of the cohort is shown in supplementary table Q. Additional adjustment for these variables resulted in slightly attenuated associations of SGLT2 inhibitors with the primary outcome, compared with the analyses without such adjustment (Sweden: hazard ratio 0.50 (0.31 to 0.81 ) and 0.41 (0.26 to 0.66$)$, respectively; Denmark: 0.55 (0.41 to 0.74 ) and 0.42 (0.32 to 0.56), respectively) In the subgroup analyses using the imputed datasets, the hazard ratios for patients with estimated glomerular filtration rate below 60 and at least 60 per $1.73 \mathrm{~m}^{2}$ body surface area were $0.47(0.30$ to 0.72 ) and $0.60(0.44$ to 0.82$)$, respectively. The hazard ratios for those with and without albuminuria were 0.45 (0.29 to 0.69 ) and 0.62 (0.43 to 0.90$)$, respectively (supplementary table $\mathrm{R}$ ).

\section{Discussion}

In this cohort study using nationwide registers in three countries, use of SGLT2 inhibitors was associated with a lower risk of the main composite outcome of serious renal events (consisting of renal replacement therapy, renal death, and hospital admission for renal events) in analyses using dipeptidyl peptidase-4 inhibitors

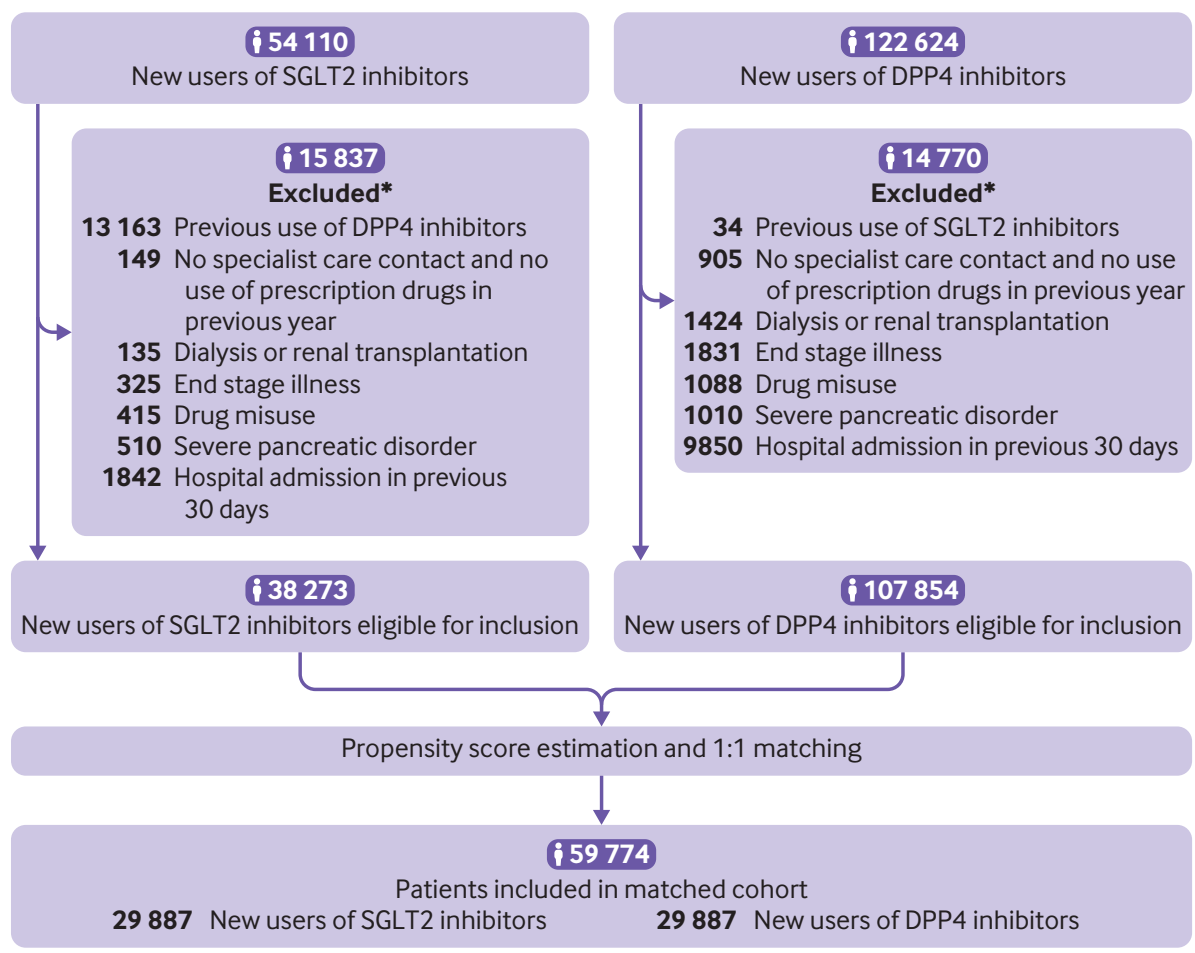

Fig 1 | Flowchart of patient inclusion in study cohort, Sweden, Denmark, and Norway. DPP4=dipeptidyl peptidase-4; SGLT2=sodium-glucose co-transporter 2. *Patients could be excluded for more than one reason 


\begin{tabular}{|c|c|c|c|}
\hline Characteristics & $\begin{array}{l}\text { SGLT2 inhibitors } \\
(\mathrm{n}=29887)\end{array}$ & $\begin{array}{l}\text { DPP4 inhibitors } \\
(n=29887)\end{array}$ & $\begin{array}{l}\text { Standardised mean } \\
\text { difference }(\%)\end{array}$ \\
\hline \multicolumn{4}{|l|}{ Country*: } \\
\hline Sweden & $9241(30.9)$ & $9241(30.9)$ & - \\
\hline Denmark & $14232(47.6)$ & $14232(47.6)$ & - \\
\hline Norway & $6414(21.5)$ & $6414(21.5)$ & - \\
\hline Male sex & $18129(60.7)$ & $18129(60.7)$ & - \\
\hline Mean (SD) age, years & $61.3(10.5)$ & $61.3(10.5)$ & 0.4 \\
\hline \multicolumn{4}{|l|}{ Age group, years: } \\
\hline $35-39$ & $744(2.5)$ & $744(2.5)$ & - \\
\hline $40-44$ & $1447(4.8)$ & $1447(4.8)$ & - \\
\hline $45-49$ & $2534(8.5)$ & $2534(8.5)$ & - \\
\hline $50-54$ & $3787(12.7)$ & $3787(12.7)$ & - \\
\hline $55-59$ & $4551(15.2)$ & $4551(15.2)$ & - \\
\hline $60-64$ & $4921(16.5)$ & $4921(16.5)$ & - \\
\hline $65-69$ & $5166(17.3)$ & $5166(17.3)$ & - \\
\hline $70-74$ & $4006(13.4)$ & $4006(13.4)$ & - \\
\hline $75-79$ & $1948(6.5)$ & $1948(6.5)$ & - \\
\hline $80-84$ & $783(2.6)$ & $783(2.6)$ & - \\
\hline \multicolumn{4}{|l|}{ Place of birth: } \\
\hline Scandinavia & $25000(83.6)$ & $25082(83.9)$ & 0.7 \\
\hline Rest of Europe & $1977(6.6)$ & $1951(6.5)$ & 0.4 \\
\hline Outside Europe & $2866(9.6)$ & $2812(9.4)$ & 0.6 \\
\hline Missing & $44(0.1)$ & $42(0.1)$ & 0.2 \\
\hline \multicolumn{4}{|l|}{ Civil status: } \\
\hline Married/living with partner & $17533(58.7)$ & $17608(58.9)$ & 0.5 \\
\hline Single & $12248(41.0)$ & $12178(40.7)$ & 0.5 \\
\hline Missing & $106(0.4)$ & $101(0.3)$ & 0.3 \\
\hline \multicolumn{4}{|l|}{ Educationt: } \\
\hline Primary/secondary school, vocational training & $18455(78.6)$ & $18556(79.1)$ & 1.1 \\
\hline Short tertiary education & $1384(5.9)$ & $1414(6.0)$ & 0.5 \\
\hline Medium or long tertiary education & $2975(12.7)$ & $2887(12.3)$ & 1.1 \\
\hline Missing & $659(2.8)$ & $616(2.6)$ & 1.1 \\
\hline \multicolumn{4}{|l|}{ Medical history: } \\
\hline Acute coronary syndrome & $2145(7.2)$ & $2039(6.8)$ & 1.4 \\
\hline Other ischaemic heart disease & $4978(16.7)$ & $4729(15.8)$ & 2.3 \\
\hline Heart failure/cardiomyopathy & $1682(5.6)$ & $1560(5.2)$ & 1.8 \\
\hline Valve disorders & $727(2.4)$ & $666(2.2)$ & 1.4 \\
\hline Stroke & $1166(3.9)$ & $1160(3.9)$ & 0.1 \\
\hline Other cerebrovascular disease & $1268(4.2)$ & $1230(4.1)$ & 0.6 \\
\hline Atrial fibrillation & $2066(6.9)$ & $1939(6.5)$ & 1.7 \\
\hline Other arrhythmia & $1258(4.2)$ & $1137(3.8)$ & 2.1 \\
\hline Coronary revascularisation in previous year & $438(1.5)$ & $436(1.5)$ & 0.1 \\
\hline Other cardiac surgery/invasive procedure in previous year & $152(0.5)$ & $126(0.4)$ & 1.3 \\
\hline Arterial disease & $1872(6.3)$ & $1871(6.3)$ & $<0.1$ \\
\hline Chronic kidney disease & $987(3.3)$ & $987(3.3)$ & - \\
\hline Other renal disease & $1664(5.6)$ & $1559(5.2)$ & 1.6 \\
\hline Diabetic complications & $8069(27.0)$ & $7828(26.2)$ & 1.8 \\
\hline Chronic obstructive pulmonary disease & $1184(4.0)$ & $1127(3.8)$ & 1.0 \\
\hline Other lung disease & $2080(7.0)$ & $2010(6.7)$ & 0.9 \\
\hline Venous thromboembolism & $693(2.3)$ & $666(2.2)$ & 0.6 \\
\hline Cancer & $1981(6.6)$ & $2002(6.7)$ & 0.3 \\
\hline Liver disease & $621(2.1)$ & $622(2.1)$ & $<0.1$ \\
\hline Rheumatic disease & $860(2.9)$ & $845(2.8)$ & 0.3 \\
\hline Psychiatric disorder & $2594(8.7)$ & $2572(8.6)$ & 0.3 \\
\hline Fracture in previous year & $497(1.7)$ & $492(1.6)$ & 0.1 \\
\hline \multicolumn{4}{|l|}{ Hospital admissions in previous year: } \\
\hline Cardiovascular causes & $1290(4.3)$ & $1322(4.4)$ & 0.5 \\
\hline Type 2 diabetes related causes & $259(0.9)$ & $266(0.9)$ & 0.3 \\
\hline Non-cardiovascular/type 2 diabetes related causes & $3890(13.0)$ & $3771(12.6)$ & 1.2 \\
\hline \multicolumn{4}{|l|}{ Outpatient contacts in previous year: } \\
\hline Cardiovascular causes & $2844(9.5)$ & $2753(9.2)$ & 1.0 \\
\hline Type 2 diabetes related causes & $5908(19.8)$ & $5714(19.1)$ & 1.6 \\
\hline Non-cardiovascular/type 2 diabetes related causes & $16894(56.5)$ & $16713(55.9)$ & 1.2 \\
\hline
\end{tabular}




\begin{tabular}{|c|c|c|c|}
\hline Characteristics & $\begin{array}{l}\text { SGLT2 inhibitors } \\
(\mathrm{n}=29887)\end{array}$ & $\begin{array}{l}\text { DPP4 inhibitors } \\
(\mathrm{n}=29887)\end{array}$ & $\begin{array}{l}\text { Standardised mean } \\
\text { difference (\%) }\end{array}$ \\
\hline \multicolumn{4}{|l|}{ Diabetes drugs in previous 6 months: } \\
\hline None & $2316(7.7)$ & $2201(7.4)$ & 1.5 \\
\hline Metformin & $24339(81.4)$ & $24541(82.1)$ & 1.8 \\
\hline Sulfonylureas & $5724(19.2)$ & $5741(19.2)$ & 0.1 \\
\hline Glucagon-like peptide 1 receptor agonists & $2607(8.7)$ & $2549(8.5)$ & 0.7 \\
\hline Insulin & $7852(26.3)$ & $7924(26.5)$ & 0.5 \\
\hline Other antidiabetics (glitazones, glinides, acarbose) & $715(2.4)$ & $717(2.4)$ & $<0.1$ \\
\hline \multicolumn{4}{|l|}{ Time since first diabetes drug, years: } \\
\hline$<1$ & 3969 (13.3) & $4029(13.5)$ & 0.6 \\
\hline $1-3$ & $3812(12.8)$ & $3865(12.9)$ & 0.5 \\
\hline$>3-5$ & $3598(12.0)$ & $3591(12.0)$ & 0.1 \\
\hline$>5-7$ & $3862(12.9)$ & $3920(13.1)$ & 0.6 \\
\hline$>7$ & $14646(49.0)$ & $14482(48.5)$ & 1.1 \\
\hline \multicolumn{4}{|l|}{ Prescription drugs in previous year: } \\
\hline ACEi/ARB & $19589(65.5)$ & $19396(64.9)$ & 1.4 \\
\hline Calcium channel blocker & $8953(30.0)$ & $8759(29.3)$ & 1.4 \\
\hline Loop diuretict & $3206(13.7)$ & $3024(12.9)$ & 2.3 \\
\hline Other diuretict & $4347(18.5)$ & $4205(17.9)$ & 1.6 \\
\hline$\beta$ blocker & $9871(33.0)$ & $9648(32.3)$ & 1.6 \\
\hline Digoxin & $639(2.1)$ & $639(2.1)$ & $<0.1$ \\
\hline Nitrate & $1964(6.6)$ & $1900(6.4)$ & 0.9 \\
\hline Platelet inhibitor & $10516(35.2)$ & $10406(34.8)$ & 0.8 \\
\hline Anticoagulant & $2198(7.4)$ & $2057(6.9)$ & 1.8 \\
\hline Lipid lowering drug & $20467(68.5)$ & $20380(68.2)$ & 0.6 \\
\hline Antidepressant & $4539(15.2)$ & $4527(15.1)$ & 0.1 \\
\hline Antipsychotic & $1164(3.9)$ & $1165(3.9)$ & $<0.1$ \\
\hline Anxiolytic hypnotic or sedative & $4654(15.6)$ & $4572(15.3)$ & 0.8 \\
\hline$\beta-2$ agonist inhalant & $2854(9.5)$ & $2772(9.3)$ & 0.9 \\
\hline Anticholinergic inhalant & $903(3.0)$ & $879(2.9)$ & 0.5 \\
\hline Glucocorticoid inhalant & $2822(9.4)$ & $2717(9.1)$ & 1.2 \\
\hline Oral glucocorticoid & $2081(7.0)$ & $2069(6.9)$ & 0.2 \\
\hline Non-steroidal anti-inflammatory drug & $7478(25.0)$ & $7475(25.0)$ & $<0.1$ \\
\hline Opioid & $5634(18.9)$ & $5560(18.6)$ & 0.6 \\
\hline \multicolumn{4}{|l|}{ No of prescription drugs in previous yeart: } \\
\hline $0-5$ & $5481(23.4)$ & $5643(24.0)$ & 1.6 \\
\hline $6-10$ & $9681(41.2)$ & $9647(41.1)$ & 0.3 \\
\hline $11-15$ & $5382(22.9)$ & $5332(22.7)$ & 0.5 \\
\hline$>15$ & $2929(12.5)$ & 2851 (12.1) & 1.0 \\
\hline
\end{tabular}

as an active comparator. Use of SGLT2 inhibitors was associated with a significantly lower risk of incident renal replacement therapy, as well as hospital admission for renal events, but not with death due to renal causes.

\section{Interpretation and comparison with previous studies}

Large clinical trials have shown that SGLT2 inhibitors can reduce the risk of advanced renal outcomes, including renal replacement therapy, and protect kidney function in patients at high risk of cardiovascular disease or established nephropathy. ${ }^{5-8} 18$ Our study adds to the knowledge about SGLT2 inhibitors and renal outcomes by using nationwide registers from three countries (Denmark (47.6\% of the patients), Sweden (30.9\%), and Norway $(21.5 \%))$ to include a large number of patients seen in routine clinical practice. Importantly, of the patients included in our study, $81 \%$ and $97 \%$ had no diagnosis of cardiovascular disease and chronic kidney disease, respectively. Although the absolute risk reduction associated with SGLT2 inhibitors was larger in patients with cardiovascular disease or chronic kidney disease, the protective association of SGLT2 inhibitors was also observed in patients without such history. The findings from this observational study complement the data from clinical trials,,$^{5-8}$ as well as our previous observational study of cardiovascular outcomes, ${ }^{19}$ and provide further support for the use of SGLT2 inhibitors across a broad range of patients with type 2 diabetes with various levels of renal function. SGLT2 inhibitors have been suggested to protect the kidney through several mechanisms, including favourable effects on renal haemodynamics and reduction of tissue inflammation and fibrosis. ${ }^{1220}$

Although the primary outcome definition used in our study did not directly correspond to the composite renal outcomes used in clinical trials (which were largely driven by albuminuria and changes in estimated glomerular filtration rate), the secondary outcome of renal replacement therapy can be compared across 


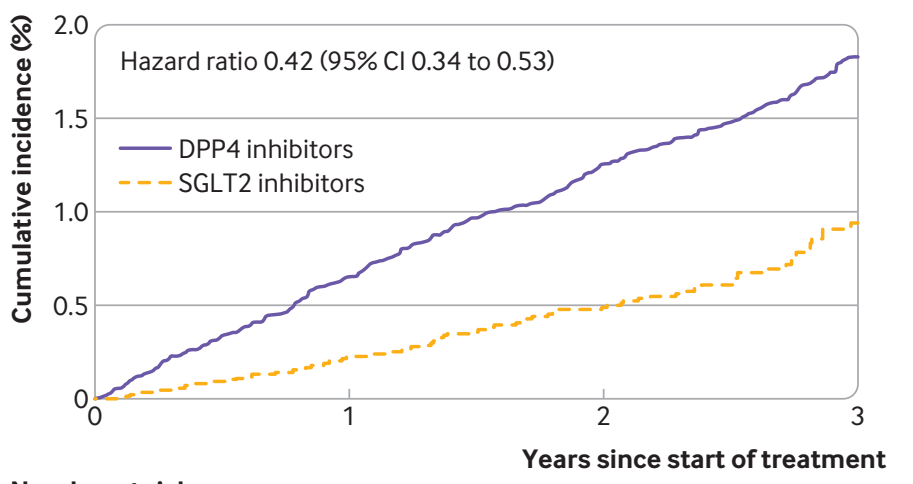

Number at risk

SGLT2 inhibitors 29887

9109

3198

DPP4 inhibitors

29887

22689

15720

9344

Fig 2 | Cumulative incidence of serious renal events in users of sodium-glucose cotransporter 2 (SGLT2) inhibitors and dipeptidyl peptidase-4 (DPP4) inhibitors

studies. For this outcome, we observed a hazard ratio of 0.32 (95\% confidence interval 0.22 to 0.47$)$ for use of SGLT2 inhibitors versus dipeptidyl peptidase-4 inhibitors, which is broadly in line with the hazard ratio versus placebo for renal replacement therapy in the EMPAREG OUTCOME trial of empagliflozin (0.45, 0.21 to 0.97$)^{7}$ and for end stage kidney disease in the DECLARE-TIMI 58 trial of dapagliflozin (0.31, 0.13 to $0.79),{ }^{8}$ whereas the hazard ratio for end stage kidney disease in the CREDENCE trial of canagliflozin was slightly higher $(0.68,0.54$ to 0.86$) .^{5}$ Dapagliflozin (66.1\% of the total follow-up time among users of SGLT2 inhibitors) and empagliflozin (32.6\%) were the most common SGLT2 inhibitors in our study population, but use of canagliflozin was rare $(1.3 \%)$. With respect to event rates, the incidence of renal replacement therapy was substantially higher in the CREDENCE trial (13.3 (canagliflozin) versus 17.7 (placebo) per 1000 person years) than in our study (0.8 (SGLT2 inhibitors) versus 2.5 (dipeptidyl peptidase-4 inhibitors) per 1000 person years), in which event rates were similar to those of the EMPAREG OUTCOME trial (1.0 (emplagliflozin) versus 2.1 (placebo) per 1000 person years). Whereas the CREDENCE trials included patients with an estimated glomerular filtration rate of 30 to less than $90 \mathrm{~mL} / \mathrm{min}$ per $1.73 \mathrm{~m}^{2}$ and albuminuria, SGLT2 inhibitors were used in a broad range of patients in routine clinical practice, although these drugs were not recommended in patients with an estimated glomerular filtration rate of less than $45 \mathrm{~mL} / \mathrm{min}$ per $1.73 \mathrm{~m}^{2}$ during the study period.

We attempted to limit the risk of confounding by using a propensity score including a wide range of patient characteristics. Moreover, we used a new user design excluding patients with a history of using any of the study drugs at cohort entry; this design removed the possibility of immortal time bias, which has been highlighted in other observational analyses of SGLT2 inhibitors. ${ }^{21}$ Another strength of the study was the use of data on glycated haemoglobin level, albuminuria, and estimated glomerular filtration rate (Sweden and Denmark), as well as blood pressure, body mass index, and smoking (Sweden) in the Swedish and Danish parts of the cohort (78.5\% of the overall cohort). When the outcome models were further adjusted for these variables in sensitivity analyses, the point estimate for the primary outcome moved from 0.41 to 0.50 in Sweden and from 0.42 to 0.55 in Denmark, indicating that the primary analyses may present slightly overestimated associations owing to confounding by these variables.

We used DPP4 inhibitors as the active comparator drug class to mitigate the risk of confounding by indication, disease severity, and unmeasured clinical characteristics. During the study period, both dipeptidyl peptidase- 4 inhibitors and SGLT2 inhibitors were recommended as second or third line glucose lowering drugs, and the two drug classes were thus used in similar clinical situations and at a similar stage of disease. ${ }^{11}$ In accordance with their neutral effect on cardiovascular outcomes and mortality, data from clinical trials in patients at high cardiovascular risk indicate that dipeptidyl peptidase- 4 inhibitors have no or limited effects on renal outcomes. ${ }^{12}$ The risk of composite renal outcomes was similar among patients receiving active treatment and placebo in the SAVOR TIMI 53 trial of saxagliptin and the CARMELINA trial of linagliptin, ${ }^{22}{ }^{23}$ although some potential benefit was indicated in exploratory analyses of albuminuria outcomes. ${ }^{23} 24$ In the EXAMINE trial, the risks of dialysis and changes in estimated glomerular filtration rate were similar in patients receiving alogliptin and placebo. ${ }^{25}$ In the TECOS trial of sitagliptin, which comprised $64.8 \%$ of the DPP4 inhibitor use in our study, the decline in estimated

\begin{tabular}{|c|c|c|c|c|c|c|}
\hline \multirow[b]{2}{*}{ Outcome } & \multicolumn{2}{|c|}{ SGLT2 inhibitors $(n=29887)$} & \multicolumn{2}{|c|}{ DPP4 inhibitors $(n=29887)$} & \multirow[b]{2}{*}{ Hazard ratio $(95 \% \mathrm{Cl})$} & \multirow[b]{2}{*}{$\begin{array}{l}\text { Absolute difference, events } \\
(95 \% \mathrm{Cl}) \text { per } 1000 \text { person years }\end{array}$} \\
\hline & Events & $\begin{array}{l}\text { Events per } 1000 \\
\text { person years }\end{array}$ & Events & $\begin{array}{l}\text { Events per } 1000 \\
\text { person years }\end{array}$ & & \\
\hline Primary outcome* & 111 & 2.6 & 360 & 6.2 & $0.42(0.34$ to 0.53$)$ & $-3.6(-4.4$ to -2.8$)$ \\
\hline \multicolumn{7}{|l|}{ Secondary outcomes: } \\
\hline Renal replacement therapy & 33 & 0.8 & 146 & 2.5 & $0.32(0.22$ to 0.47$)$ & $-1.7(-2.2$ to -1.2$)$ \\
\hline Death from renal causest & 5 & 0.2 & 11 & 0.2 & $0.77(0.26$ to 2.23$)$ & $-0.1(-0.2$ to 0.1$)$ \\
\hline Hospital admission for renal events & 85 & 2.0 & 285 & 4.9 & $0.41(0.32$ to 0.52$)$ & $-2.9(-3.6$ to -2.2$)$ \\
\hline
\end{tabular}




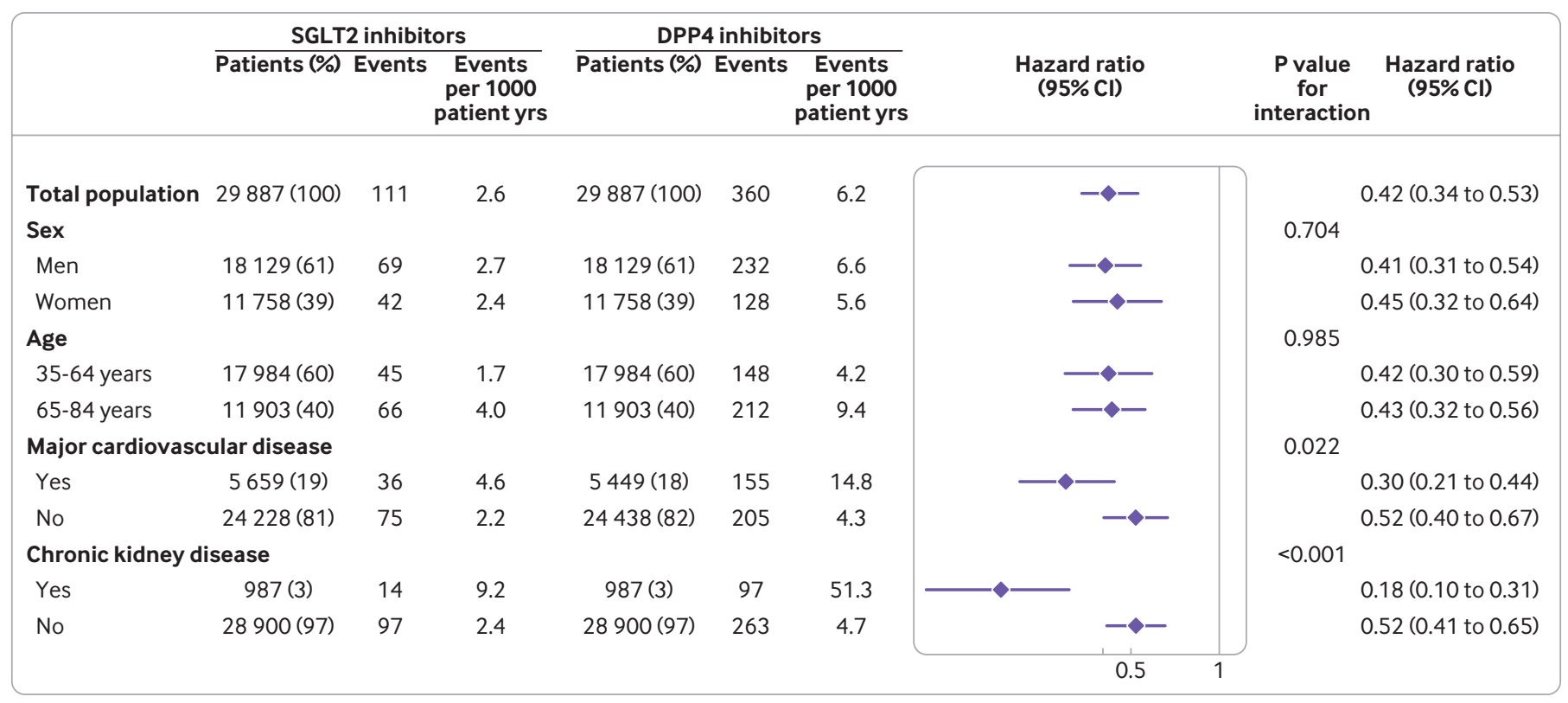

Fig 3 | Subgroup analyses of serious renal events among users of sodium-glucose co-transporter 2 (SGLT2) inhibitors compared with users of dipeptidyl peptidase-4 (DPP4) inhibitors

glomerular filtration rate was clinically similar in patients receiving active treatment and placebo. ${ }^{26}$ If dipeptidyl peptidase- 4 inhibitors have a protective effect on renal outcomes, the associations observed in our study may represent an underestimation of the effects of SGLT2 inhibitors. However, this would not change the overall interpretation of our analyses, and the study would still present a head-to-head comparative effectiveness analysis of SGLT2 inhibitors and dipeptidyl peptidase- 4 inhibitors. Furthermore, comparative effectiveness analyses of other drug classes with demonstrated renoprotective effects, ${ }^{12}$ such as glucagon-like peptide-1-receptor-agonists, remain a topic for future investigation.

\section{Limitations of study}

Our study has limitations. Firstly, the definition of exposure was based on filled prescriptions; low adherence may bias the results towards the null. Secondly, the study was conducted in Scandinavia, and its generalisability to other populations and healthcare systems is unknown. Thirdly, although high sensitivity and positive predictive values have been observed for procedure codes and diagnoses recorded in Scandinavian health registers, ${ }^{27}$ data on covariates and outcomes in these registers may be incomplete or misclassified. With respect to the specific codes used for the outcome definition in our study, validation studies in the Scandinavian setting have not been conducted. ${ }^{27} 28$ Outcome misclassification could have introduced bias in our analyses; however, such misclassification is not likely to be different in patients receiving SGLT2 inhibitors versus dipeptidyl peptidase-4 inhibitors. Finally, although we used an active comparator, new user design and estimated a propensity score to control for a large number of patient characteristics, this was an observational study and the risk of unmeasured confounding cannot be ruled out.

\section{Conclusions}

In this analysis of nationwide registers from three countries, use of SGLT2 inhibitors, compared with dipeptidyl peptidase-4 inhibitors, was associated with a reduced risk of serious renal events. Complementing data from clinical trials, this study provides further support for the use of SGLT2 inhibitors in a broad range of patients with type 2 diabetes.

\section{AUTHOR AFFILIATIONS}

${ }^{1}$ Clinical Epidemiology Division, Department of Medicine, Solna, Karolinska Institutet, Stockholm, Sweden

${ }^{2}$ Department of Epidemiology Research, Statens Serum Institut, Copenhagen, Denmark

${ }^{3}$ Department of Clinical Medicine, University of Copenhagen, Copenhagen, Denmark

${ }^{4}$ Department of Medicine, Stanford University School of Medicine, Stanford, CA, USA

${ }^{5}$ Department of Molecular and Clinical Medicine, Institute of Medicine, University of Gothenburg, Gothenburg, Sweden

${ }^{6}$ Swedish National Diabetes Register, Västra Götalandsregionen, Gothenburg, Sweden

${ }^{7}$ Health Metrics, Department of Public Health and Community Medicine, Sahlgrenska Academy, University of Gothenburg, Gothenburg, Sweden

${ }^{8} \mathrm{~K}$ G Jebsen Center for Genetic Epidemiology, Department of Public Health and Nursing, Faculty of Medicine and Health Science, Norwegian University of Science and Technology, Trondheim, Norway

${ }^{9}$ HUNT Research Centre, Faculty of Medicine, Norwegian University of Science and Technology, Levanger, Norway

${ }^{10}$ Division of Health Data and Digitalisation, Norwegian Institute of Public Health, Oslo, Norway

Contributors: BP and PU had full access to all the data in the study and take responsibility for the integrity of the data and the accuracy of the data analysis. BP, VW, HS, and PU were responsible for the concept and design of the study. All authors were involved in the acquisition, analysis, or interpretation of data. BP and PU drafted the manuscript, and all authors revised it critically for important intellectual content. VW did the statistical analysis. BP and PU obtained funding. BP 
supervised the study. The corresponding author attests that all listed authors meet authorship criteria and that no others meeting the criteria have been omitted. BP and PU are the guarantors.

Funding: BP was supported by an investigator grant from the Strategic Research Area Epidemiology programme at Karolinska Institutet. PU was supported by grants from the Swedish Heart-Lung Foundation and the Swedish Society for Medical Research. The study was conducted with research grant support from the Swedish Cancer Society, the Nordic Cancer Union, and the Novo Nordisk Foundation. No specific funding was received for the study. The funding sources had no role in the design and conduct of the study; collection, management, analysis, and interpretation of the data; preparation, review, or approval of the manuscript; and the decision to submit the manuscript for publication.

Competing interests: All authors have completed the ICMJE uniform disclosure form at www.icmje.org/coi disclosure.pdf and declare: no support from any organisation for the submitted work other than those described above; $\mathrm{C}$ ) has received personal fees from Pfizer and Bayer outside the submitted work; BE has received personal fees from Amgen, AstraZeneca, Boerhringer Ingelheim, Eli Lilly, Merck Sharp and Dohme, Mundipharma, Navamedic, Novo Nordisk, and RLS Global outside the submitted work and grants from Sanofi outside the submitted work; SG has received lecture fees and research grants from AstraZeneca, Boerhringer Ingelheim, Eli Lilly, Merck Sharp and Dohme, Novo Nordisk, and Sanofi outside of the submitted work; AMS has received consulting fees from Celgene and has been employed at IQVIA outside the submitted work.

Ethical approval: The study was approved by the Regional Ethics Committee in Stockholm, Sweden, and the Regional Committee for Medical and Health Research Ethics (REC Central), Norway. In Denmark, ethics approval is not required for register based research.

Data sharing: No additional data available.

Transparency declaration: The lead author affirms that the manuscript is an honest, accurate, and transparent account of the study being reported; that no important aspects of the study have been omitted; and that any discrepancies from the study as planned (and, if relevant, registered) have been explained.

Dissemination to participants and related patient and public communities: The findings of this study will be disseminated via the media departments and websites of the authors' institutes.

This is an Open Access article distributed in accordance with the Creative Commons Attribution Non Commercial (CC BY-NC 4.0) license, which permits others to distribute, remix, adapt, build upon this work non-commercially, and license their derivative works on different terms, provided the original work is properly cited and the use is noncommercial. See: http://creativecommons.org/licenses/by-nc/4.0/.

1 Thomas MC, Cooper ME, Zimmet P. Changing epidemiology of type 2 diabetes mellitus and associated chronic kidney disease. Nat Rev Nephrol 2016;12:73-81. doi:10.1038/ nrneph.2015.173

2 Lewis EJ, Hunsicker LG, Bain RP, Rohde RD, The Collaborative Study Group. The effect of angiotensin-converting-enzyme inhibition on diabetic nephropathy. N Engl J Med 1993;329:1456-62. doi:10.1056/NEJM199311113292004

3 Lewis EJ, Hunsicker LG, Clarke WR, et al, Collaborative Study Group. Renoprotective effect of the angiotensin-receptor antagonist irbesartan in patients with nephropathy due to type 2 diabetes. $N$ Engl I Med 2001;345:851-60. doi:10.1056/NEJMoa011303

4 Brenner BM, Cooper ME, de Zeeuw D, et al, RENAAL Study Investigators. Effects of losartan on renal and cardiovascular outcomes in patients with type 2 diabetes and nephropathy. N Engl Med 2001:345:861-9 doi:10.1056/NEJMoa011161

5 Perkovic V, Jardine MJ, Neal B, et al, CREDENCE Trial Investigators. Canagliflozin and renal outcomes in type 2 diabetes and nephropathy. N Engl J Med 2019;380:2295-306. doi:10.1056/ NEJMoa1811744

6 Perkovic V, de Zeeuw D, Mahaffey KW, et al. Canagliflozin and renal outcomes in type 2 diabetes: results from the CANVAS Program randomised clinical trials. Lancet Diabetes Endocrinol 2018;6:691704. doi:10.1016/S2213-8587(18)30141-4

7 Wanner C, Inzucchi SE, Lachin JM, et al, EMPA-REG OUTCOME Investigators. Empagliflozin and Progression of Kidney Disease in Type 2 Diabetes. N Engl / Med 2016;375:323-34. doi:10.1056/ NEJMoa1515920

8 Mosenzon O, Wiviott SD, Cahn A, et al. Effects of dapagliflozin on development and progression of kidney disease in patients with type 2 diabetes: an analysis from the DECLARE-TIMI 58 randomised trial.
Lancet Diabetes Endocrinol 2019;7:606-17. doi:10.1016/S2213 8587(19)30180-9

9 Beekman-Hendriks WL, Thuresson M, Pignot M, et al. How Representative are the Patients Included in the CV outcome Trials with SGLT2 Inhibitors of a General Type 2 Diabetes Population? A Large European Observational study. Diabetes Obes Metab 2018;1:1-7

10 Lund JL, Richardson DB, Stürmer T. The active comparator, new user study design in pharmacoepidemiology: historical foundations and contemporary application. Curr Epidemiol Rep 2015;2:221-8. doi:10.1007/s40471-015-0053-5

11 Inzucchi SE, Bergenstal RM, Buse JB, et al. Management of hyperglycemia in type 2 diabetes, 2015: a patient-centered approach: update to a position statement of the American Diabetes Association and the European Association for the Study of Diabetes. Diabetes Care 2015:38:140-9. doi:10.2337/dc14-2441

12 Muskiet MHA, Wheeler DC, Heerspink HJL. New pharmacological strategies for protecting kidney function in type 2 diabetes. Lancet Diabetes Endocrinol 2019;7:397-412.

13 D’Agostino RB, Rubin DB. Estimating and Using Propensity Scores with Partially Missing Data. J Am Stat Assoc 2000;95:749-59. doi:10. 1080/01621459.2000.10474263

14 Austin PC. Some methods of propensity-score matching had superio performance to others: results of an empirical investigation and Monte Carlo simulations. Biom J 2009;51:171-84. doi:10.1002/ bimi.200810488

15 Rassen JA, Shelat AA, Myers J, Glynn RJ, Rothman KJ, Schneeweiss $\mathrm{S}$. One-to-many propensity score matching in cohort studies. Pharmacoepidemiol Drug Saf 2012;21(Suppl 2):69-80. doi:10.1002/pds.3263

16 Grams ME, Plantinga LC, Hedgeman E, et al, CDC CKD Surveillance Team. Validation of CKD and related conditions in existing data sets: A systematic review. Am J Kidney Dis 2011;57:44-54. doi:10.1053/j. ajkd.2010.05.013

17 Harel O, Zhou X-H. Multiple imputation: review of theory, implementation and software. Stat Med 2007;26:3057-77. doi:10.1002/sim.2787

18 Neuen BL, Young T, Heerspink HJL, et al. SGLT2 inhibitors for the prevention of kidney failure in patients with type 2 diabetes: a systematic review and meta-analysis. Lancet Diabetes Endocrinol 2019;2:1-10. doi:10.1016/S2213-8587(19)30256-6

19 Pasternak B, Ueda P, Eliasson B, et al. Use of sodium glucose cotransporter 2 inhibitors and risk of major cardiovascular events and heart failure: Scandinavian register based cohort study. BMJ 2019;366:14772. doi:10.1136/bmj.14772

20 Heerspink HJL, Perkins BA, Fitchett DH, Husain M, Cherney DZI. Sodium Glucose Cotransporter 2 Inhibitors in the Treatment of Diabetes Mellitus: Cardiovascular and Kidney Effects, Potentia Mechanisms, and Clinical Applications. Circulation 2016;134:75272. doi:10.1161/CIRCULATIONAHA.116.021887

21 Suissa S. Lower Risk of Death With SGLT2 Inhibitors in Observational Studies: Real or Bias? Diabetes Care 2018;41:6-10. doi:10.2337 dc17-1223

22 Scirica BM, Bhatt DL, Braunwald E, et al, SAVOR-TIMI 53 Steering Committee and Investigators. Saxagliptin and cardiovascular outcomes in patients with type 2 diabetes mellitus. N Engls Med 2013;369:1317-26. doi:10.1056/NEJMoa1307684

23 Rosenstock J, Perkovic V, Johansen OE, et al, CARMELINA Investigators. Effect of Linagliptin vs Placebo on Major Cardiovascula Events in Adults With Type 2 Diabetes and High Cardiovascular and Renal Risk: The CARMELINA Randomized Clinical Trial. JAMA 2019;321:69-79. doi:10.1001/jama.2018.18269

24 Mosenzon O, Leibowitz G, Bhatt DL, et al. Effect of Saxagliptin on Renal Outcomes in the SAVOR-TIMI 53 Trial. Diabetes Care 2017;40:69-76. doi:10.2337/dc16-0621

25 White WB, Cannon CP, Heller SR, et al, EXAMINE Investigators. Alogliptin after acute coronary syndrome in patients with type 2 diabetes. N Engl J Med 2013;369:1327-35. doi:10.1056/ NEJMoa1305889

26 Cornel JH, Bakris GL, Stevens SR, et al, TECOS Study Group. Effect of Sitagliptin on Kidney Function and Respective Cardiovascular Outcomes in Type 2 Diabetes: Outcomes From TECOS. Diabetes Care 2016:39:2304-10. doi:10.2337/dc16-1415

27 Schmidt M, Schmidt SAJ, Sandegaard JL, Ehrenstein V, Pedersen L, Sørensen HT. The Danish National Patient Registry: a review of content, data quality, and research potential. Clin Epidemiol 2015;7:449-90. doi:10.2147/CLEP.S91125

28 Ludvigsson JF, Andersson E, Ekbom A, et al. External review and validation of the Swedish national inpatient register. BMC Public Health 2011;11:450. doi:10.1186/1471-2458-11-450

Web appendix: Supplementary material 